\title{
Avaliação de risco pelo método Hazard Rating Number em setor de indústria produtora de biodiesel em Porto Nacional/TO
}

Os setores industriais empregam dezenas de trabalhadores para execução de atividades provedoras de riscos eminentes, tais como utilização e manutenção de máquinas, trabalhos em altura e trabalhos em confinamento. Saúde, condições de trabalho e acidentes são preocupações dos trabalhadores desde o início do processo de industrialização, e com base essas informações o presente trabalho faz menção a um procedimento de avaliação de risco juntamente com um técnico de segurança identificando as áreas mais agravantes no processo fabril. Teve como objetivo levantar os principais riscos do setor de manutenção de uma empresa produtora de biodiesel utilizando as técnicas de análise de risco: Hazard Rating Number e Análise Preliminar de Risco. Os resultados obtidos pelo uso das técnicas de análise de risco permitiram quantificar o grau de perigo das atividades referentes à manutenção de fábrica, quanto apresentar sugestões para que estes sejam eliminados ou amenizados.

Palavras-chave: Análise de Riscos; Segurança; Hazard Rating.

\section{Risk assessment by Hazard Rating Number method in biodiesel industry sector in Porto Nacional/TO}

\begin{abstract}
Industrial sectors employ dozens of workers to perform imminent hazards, such as the use and maintenance of machinery, work at heights, and work in confinement. Health, working conditions and accidents are workers' concerns since the beginning of the industrialization process, and based on this information the present work mentions a risk assessment procedure together with a safety technician identifying the most aggravating areas in the manufacturing process. . It aimed to address the main risks in the maintenance sector of a biodiesel producer using the risk analysis techniques: Hazard Rating Number and Preliminary Risk Analysis. The results obtained through the use of risk analysis techniques allowed quantifying the degree of danger of the activities related to the factory maintenance, as well as presenting suggestions for their elimination or mitigation.
\end{abstract}

Keywords: Risk analysis; Safety; Hazard Rating.

Topic: Engenharia Civil

Reviewed anonymously in the process of blind peer.
Received: 22/03/2019

Approved: 12/07/2019
Maykon Douglas Martins Paulino
Referencing this:

PAULINO, M. D. M.. Avaliação de risco pelo método Hazard Rating Number em setor de indústria produtora de biodiesel em Porto Nacional/TO. Inventionis, v.1, n.2, p.9-18, 2019. DOI: http://doi.org/10.6008/CBPC2674-6395.2019.002.0002 


\section{INTRODUÇÃO}

O presente trabalho tem como objetivo auxiliar aos membros, comissões de fábrica, SESMT, e aos trabalhadores, sobre os perigos pré-existentes no âmbito do polo industrial, viabilizando e identificando medidas preventivas para minimizar a incidência de acidente de acordo com os métodos de análise de risco. Segundo a Organização Mundial de Saúde os acidentes e doenças do trabalho são responsáveis por mais de 120 milhões de lesões e pelo menos 220 mil mortes por ano no plano mundial. Os imprevistos são referentes a situações adversas de trabalho, como presença de maquinários, organização de trabalho, tarefas, condições do ambiente e sobrecargas de colaboradores (OMS, 2010).

O Ministério do Trabalho e Emprego (MTE) afirma que grande parte das eventualidades são previsíveis, ou seja, grande parte dos acidentes podem ser evitados. Essas eventualidades podem ser analisadas ante seus fatores de risco. O conhecimento desses fatores aumenta significativamente as possibilidades de previdência (BRASIL, 2009).

O desenvolvimento de práticas de gestão de segurança com base as normas regulamentadoras fazem com que essa probabilidade de risco diminua se fazendo necessária no local de trabalho e contribuindo para descriminação dos danos possíveis. A implantação das NR proporciona que o engenheiro de segurança deixe de ser apenas um superintendente e passe a ser um planejador e gestor em controle de risco. As normas foram anexadas à consolidação das leis do trabalho se tornando obrigatoriedade perante segurança e saúde do trabalhador dando mais responsabilidade as empresas e empregadores (BRASIL, 1943).

As normas regulamentadoras auxiliam o profissional de segurança na gestão de trabalhos em riscos, estabelecendo instruções e parâmetros de acordo com cada função desempenhada. Assim o trabalho se dispõe em apresentar um sistema de gerenciamento de segurança de processo, realizado no setor de manutenção de uma indústria química produtora de biodiesel do estado Tocantins, planejando técnicas de análise de risco para eliminar os riscos no ambiente de trabalho e representar a metodologia de Hazard Rating Number (HRN) juntamente com as técnicas Análise Preliminar de Risco (APR) e aplicação de checklist. É um método simples sem complexidade, que atende as disponibilidades da NBR12 e auxilia a gestão de segurança da empresa possibilitando saber o grau de risco de determinada atividade produtiva.

\section{REVISÃO TÉORICA}

\section{Segurança no trabalho}

Segundo a pesquisa de Sousa (2006) são poucos proprietários empresariais que se preocupam com segurança industrial, desconhecendo a severidade dos custos decorrente de acidentes de trabalho. 0 ministério do trabalho afirma que os acidentes de trabalho por falta de segurança, faz parte sim da gestão industrial. Os incidentes geram perca na qualidade de produção, multas e embargos (BRASIL, 2009).

O ministério de previdência social comprova que entre as décadas de 70 e 2000, houve declínio em acidentes de trabalho e fatalidades atribuídos pela organização de gestão de segurança. A importância da implantação de gerenciamento de segurança evidencia estruturas e técnicas que soluciona os riscos 
específicos a atividades industriais (BRASIL, 2009).

Cardella (2010), considera segurança no trabalho como principal função administrativa de todo e qualquer complexo industrial, visando redução de danos e perdas. Nas décadas mais recentes o desafio dos profissionais de segurança resume em promover a elaboração de técnicas de conscientização da prática frequente de comportamento seguro no ambiente de trabalho, promover aperfeiçoamento das atividades propostas e elaborar técnicas de motivação para os colaboradores prezarem pela sua integridade física (CARDELLA, 2010).

A responsabilidade pelo resguardo e garantia de segurança do colaborador cabe ao serviço especializado em engenharia de segurança e medicina do trabalho (SESMT) cujo regime de trabalho é dirigido pela consolidação de leis do trabalho (BRASIL, 1943). Segundo NR4 toda empresa que possua certa quantidade de colaboradores dirigidos pela consolidação do trabalho, devera conforme seu grau de risco, constituir o serviço especializado em engenharia de segurança e medicina no trabalho (SESMT).

O serviço especializado, juntamente com a empresa deve dispor de equipamentos de segurança gratuitamente adequados ao risco de trabalho. A NR6 trata sobre uso e disponibilização de EPI- equipamento de proteção individual que resguarda o trabalhador de um dano maior na ocorrência de acidente (ABNT, 2013). De acordo ministério do trabalho e emprego - MTE existem 36 normas regulamentadoras que auxiliam na gestão de segurança do trabalho. Obedecendo as obrigatoriedades das normas, é possível diminuir os riscos.

\section{Riscos}

Segundo Cicco et al. (2003), sempre existiu risco em qualquer atividade elaborada pelo homem no seu setor de trabalho, apenas não existia estudo e análise detalhada sobre os possíveis danos e perigos ocupacionais. A viabilidade de dano consequente de atividades profissionais é classificada como risco ocupacional. O risco pode ser dimensionado em função da probabilidade e da gravidade do dano possível e definido pela exposição de pessoas a perigos (CARDELLA, 2010).

Os riscos operacionais têm início nas atividades nocivas e perigosas de trabalho, podendo chegar a fatalidade. Mauro et al. (2004) destaca que a incidência de acidentes de trabalho e mortalidade não é tão baixa, ocupam destaque uma vez que se apresentam como a concretização dos agravos à sua saúde em decorrência da atividade produtiva.

Os perigos trabalhistas podem ser mais variados no setor industrial. Uma pesquisa feita em 2014 verifica os principais acidentes e doenças ocupacionais voltados ao setor metalúrgico de Piracicaba - SP. Reconheceu que tanto homem como mulher estão expostos a grandes incidências de acidentes, onde membros superiores, inferiores, mãos e olhos são os mais afetados pelas ocorrências.

Considera-se fator de risco, toda circunstância que aumenta a probabilidade de ocorrência do elemento indesejado. Segundo Mauro et al. (2004), os fatores de risco são definidos em cinco grupos. Riscos físicos- agressões ou situações adversas, químicos- elemento químico líquido ou gasoso, biológicosmicrorganismo hospitalares e laboratoriais, ergonômicos- gestão de trabalho, Acidentes-organização 
sinalização e proteção das máquinas.

Tabela 1: Característica de riscos.

\begin{tabular}{|l|l|l|l|l|}
\hline $\begin{array}{l}\text { Riscos } \\
\text { Físicos }\end{array}$ & $\begin{array}{l}\text { Riscos } \\
\text { Químicos }\end{array}$ & $\begin{array}{l}\text { Riscos } \\
\text { Biológicos }\end{array}$ & $\begin{array}{l}\text { Riscos } \\
\text { Ergonômicos }\end{array}$ & $\begin{array}{l}\text { Riscos de } \\
\text { Acidentes }\end{array}$ \\
\hline Ruídos & Poeira & Vírus & Esforço físico intenso & Arranjo físico inadequado \\
\hline Vibrações & Gases & Bactérias & $\begin{array}{l}\text { Levantamento e transporte } \\
\text { manual de peso }\end{array}$ & $\begin{array}{l}\text { Máquina e equipamento sem } \\
\text { proteção }\end{array}$ \\
\hline Radiações & Vapores & Protozoários & Postura inadequada & $\begin{array}{l}\text { Ferramenta inadequada ou } \\
\text { defeituosa }\end{array}$ \\
\hline Frio & Neblinas & Fungos & Controle rígido de produtividade & lluminação inadequada \\
\hline Calor & $\begin{array}{l}\text { Produtos químicos } \\
\text { em geral }\end{array}$ & Parasitas & Jornada de trabalho & Eletricidade \\
\hline $\begin{array}{l}\text { Pressões } \\
\text { anormais }\end{array}$ & Nevoas & Bacilos & Trabalho em turno noturno & $\begin{array}{l}\text { Probabilidade de explosão ou } \\
\text { incêndio }\end{array}$ \\
\hline Umidade & Fumaça & & Imposição de ritmo excessivo & Animais peçonhentos \\
\hline
\end{tabular}

Fonte: Ministério do Trabalho (2009).

Para lidar com os riscos foi desenvolvido estudos de gerenciamento de perigo, cujo objetivo é analisar e identificar eventos futuros incertos que podem influenciar no desenvolvimento de atividades de trabalho (CARDELLA, 2010).

\section{Avaliação de risco}

Gerenciamento de risco é um processo que minimiza as probabilidades e consequências de eventos negativos da realização de trabalho (DOONAN, 2001). A gestão de risco envolve avaliação e tomada de decisões que permite elaborar recurso para eliminar, evitar ou diminuir os riscos. É importante para as empresas ter ampla visão sobre o sistema de gerenciamento de risco, contribui para identificação das vulnerabilidades do sistema industrial e seu processo de produção. Como se trata de indústria química, a gestão de risco inclui todos os aspectos de avaliação de forma a enquadrá-la em nível aceitável de exposição (LAPA et al., 2011).

As unidades de produção química possuem grande diversidade de produtos inflamáveis, assim promove acidentes e atividades que devem ser analisados em função de seu modo de operação (FERREIRA, 2007). No trabalho de Oliveira (2008), foi citado e desenvolvido os métodos APR e HAZARD para gestão de acidentes ocorridos em uma empresa petrolífera no Brasil, com propostas de neutralização dos perigos.

Oliveira (2008) mostra a prática de gestão de risco na produção de petróleo da Petrobrás representando as metodologias de análise de risco aplicadas no seu projeto minimizando as fatalidades no processo e servindo como referência para outras empresas inflamáveis. Independente da metodologia de análise de risco, é preciso seguir uma linha de estudo, tal como: identificação das consequências e identificação dos perigos. A sondagem de dado pode ser realizada através de atividade em campo, análise preliminar de risco, ou listas de verificação (CICCO et al., 2003).

Análise preliminar de risco - APR se deu origem na área militar para revisão de sistemas de misseis. É uma técnica de análise que consiste em identificar eventos perigosos caracterizado como medida preventiva que antecipa o início de operação do sistema. Essa metodologia levanta para cada risco identificado, as causas e efeitos de eventos indesejados sobre os colaboradores e meio ambiente. Leva em 
consideração os riscos inerentes ao local e os riscos oriundos da execução de serviço (CICCO et al., 2003).

O método de Hazard analisado por Oliveira (2008) se tornou conhecido a partir do artigo da revista Safety (1990) pelo autor Chris Steel, assim como APR essa metodologia também é caracterizada como procedimento de análise de risco, determina estimativa de risco para cada perigo encontrado (SANTOS, 2018).

Hazard Rating Number é uma metodologia quantitativa que estima possível ocorrência de dano e a provável magnitude de efeito adverso. Ressaltam os aspectos de segurança do trabalhador, saúde, valores patrimoniais e financeiros (PINTO, 2014). A pesquisa de Pinto (2014), mostra toda metodologia de HRN dentro da gestão de segurança no trabalho. Procedimento regido pela $\mathrm{Nr} 12$, frequente e usado em análise de risco em máquinas, classificando os riscos baseados em diversos fatores e parâmetros.

A ferramenta HRN ou seja, número de avaliação de perigos é usado e reconhecido mundialmente nos sistemas industriais. Para determinada situação de risco, o número de HRN é o produto de quatro parâmetros: FE (Frequência de exposição), GPL (Grau de possível dano) PO (Probabilidade de ocorrência) NP (Número de pessoas expostas), que possibilita criar lista de prioridades, do maior para o menor risco, o que auxilia na tomada de decisões (SANTOS, 2018).

Método eficaz que procura pontos vulneráveis e avalia as saídas propostas, determinando seus riscos através da norma regulamentadora Nr12 em seu processo de análise. A norma trata sobre definições de referência técnica de prevenção de acidentes e doenças no trabalho, fases de projeto e utilização de máquinas e equipamentos novos ou usados. Define princípios fundamentais e medidas de proteção para garantir a saúde e integridade física do trabalhador (ABNT, 2013).

Todo trabalho que foge da rotina normal, desprovido de procedimento específico, deve ser analisado o risco, considerando a quantidade, a forma de armazenamento, o local em que será depositado e como será a sua movimentação (PINTO, 2014). O procedimento de Hazard facilita o processo de avaliação sendo assim importante para a pesquisa em questão.

\section{METODOLOGIA}

\section{A Área de Estudo}

A metodologia do trabalho consiste em empregar as técnicas de análise de risco Hazard Ranking Number no setor de manutenção de indústria química situada no estado do Tocantins. A análise dos resultados tem por finalidade propor melhorias no setor de trabalho permitindo descrever e analisar a complexidade de um eventual problema. A pesquisa permite encontrar e prevenir fenômenos de acidente no trabalho no seu local de situação.

A empresa é situada no interior do estado do Tocantins, considerada como grande produtora de biodiesel e outros derivados (glicerina, óleo de soja, Borra) provenientes do processo de produção. O biodiesel é derivado de ácidos provenientes de óleo vegetal extraído da soja, produzido e comercializado a partir da resolução n42/2004 - legislação Agência Nacional do Petróleo que prevê a determinação de suas 
características. Empresa $100 \%$ brasileira com mais de 1800 colaboradores comprometidos na produção e qualidade do produto (Figura 1).

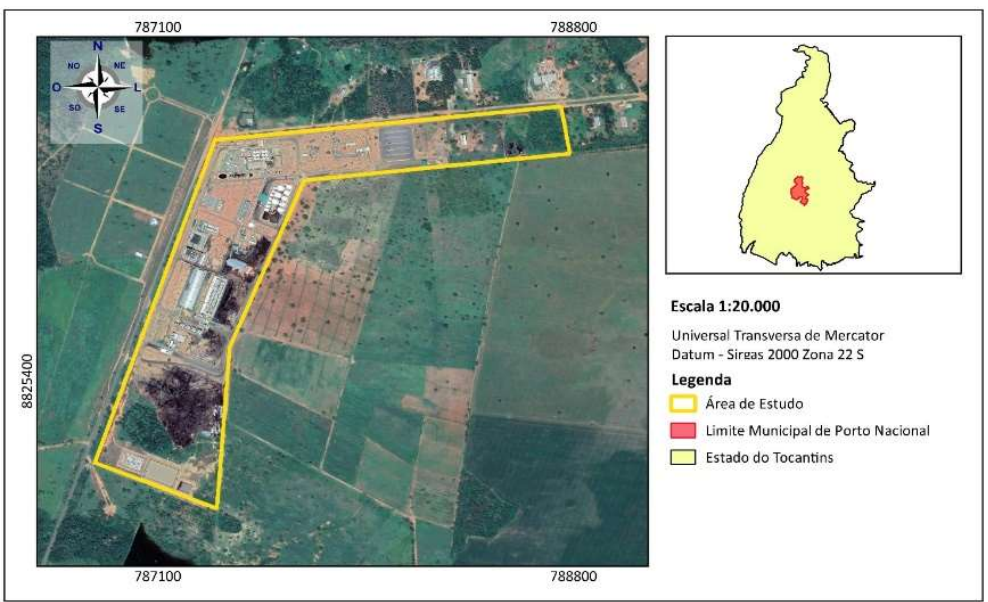

Figura 1: Mapa da área estudada.

O trabalho é voltado especificamente ao setor de manutenção exposto aos riscos referentes à produção e funcionamento da fábrica. A pesquisa foi realizada in loco acompanhada pelo profissional técnico responsável pela área. A metodologia aplicada no processo segue o método de HRN, procedimento de quantificação de risco. Traduz número de avaliação de perigos, classifica o risco de raro a extremo de acordo com seus parâmetros. Avalia o perigo e elabora padrão de segurança para o trabalho, consegue ordenar e elaborar plano de ação para diminuição dos riscos existentes.

\section{Cálculo do HRN}

O HRN é expresso em valor numérico que classifica o grau de risco do setor. O cálculo do HRN neste estudo foi feito aplicando a seguinte equação:

$$
H R N=P O \times F E x G P L \times N P
$$

PO: Probabilidade de ocorrência; FE: Frequência de exposição ao risco; GPL: Grau de um possível dano; NP: Número de pessoas expostas ao risco; Cada parâmetro mencionado acima representa a variável de cálculo para encontrar o HRN ou item avaliado. A Tabela 1 abaixo foi utilizada para determinar o critério de verificação do método.

Tabela 2: Critério de verificação do HRN.

\begin{tabular}{|l|l|}
\hline HRN & RISCOS \\
\hline $0-1$ & Raro \\
\hline $1-5$ & Baixo \\
\hline $5-50$ & Atenção \\
\hline $50-100 I$ & Significativo \\
\hline $100-500$ & Alto \\
\hline$>500$ & Extremo \\
\hline
\end{tabular}


Alto: indica risco que necessita de medidas de segurança em curto prazo;

Extremo: indica risco que necessita de medidas de segurança imediata.

Com base os parâmetros estabelecidos e mediante os resultados encontrados, podemos determinar os níveis de risco do trabalho avaliado pelo HRN, sendo ele com máquinas ou equipamentos de trabalho. Para cada item deve se utilizar as classificações que seguem na Tabela 3.

Tabela 3: Classificação dos parâmetros.

\begin{tabular}{|l|l|l|l|l|l|l|l|}
\hline \multicolumn{2}{|c|}{$\begin{array}{c}\text { Probabilidade de ocorrência } \\
\text { (PO) }\end{array}$} & \multicolumn{2}{c|}{$\begin{array}{c}\text { Grau de possível dano } \\
\text { (GPL) }\end{array}$} & \multicolumn{1}{c|}{$\begin{array}{c}\text { Frequência de exposição } \\
\text { (FE) }\end{array}$} & \multicolumn{2}{c|}{$\begin{array}{c}\text { Número pessoas sob risco } \\
\text { (NP) }\end{array}$} \\
\hline Quase impossível & 0,033 & Arranhão/Escoriação & 0,1 & Anualmente & 0,5 & $1-2$ Pessoas & 1 \\
\hline Altamente improvável & 1 & Corte/Enfermidade Leve & 0,5 & Mensalmente & 1 & $3-7$ Pessoas & 2 \\
\hline Improvável & 1,5 & Fratura leve de ossos & 1 & Semanalmente & 1,5 & $8-15$ Pessoas & 4 \\
\hline Possível & 2 & Fratura grave de ossos & 2 & Diariamente & 2,5 & $16-50$ Pessoas & 8 \\
\hline Alguma chance & 5 & Perda de 1 ou 2 dedos & 4 & Em termos de hora & 4 & Mais de 50 pessoas & 12 \\
\hline Provável & 8 & Amputação & 8 & Constantemente & 5 & & \\
\hline Muito provável & 10 & $\begin{array}{l}\text { Enfermidade } \\
\text { permanente }\end{array}$ & 12 & & & & \\
\hline Certeza & 15 & Fatalidade & 15 & & & & \\
\hline
\end{tabular}

O método de HRN obedece a norma NR12 onde se considera as características das máquinas e equipamentos, do processo, apreciação de risco e estado da técnica. O método pode ser adaptado a qualquer avaliação de análise de risco. O cálculo do HRN foi feito de acordo os riscos potenciais de execução de trabalho nas linhas da centrifuga, realizada pelo operador de manutenção. Foi realizada APR no local da tarefa juntamente com o profissional de segurança no trabalho, e por meio de checklist foram avaliados os riscos potenciais da tarefa, as medidas preventivas de controle ou recomendações de segurança, e descrição da atividade a ser elaborada.

\section{Execução da investigação}

Abertura de Autorização de Trabalho em Risco (ATR) para realização da tarefa contendo aprovação do supervisor de manutenção, responsável técnico de segurança, e executantes do trabalho, assim apresentado na Tabela 05. O documento especifica quais os riscos presentes na execução da tarefa e quais as contramedidas que devem ser obedecidas pelo colaborador. Aponta os riscos existentes nas tarefas e as medidas de controle adotadas para a sua eliminação, e ou neutralização.

Tabela 4: Avaliação de Perigos e Riscos.

\begin{tabular}{|c|c|c|c|c|c|c|c|c|c|c|c|c|c|c|c|c|c|c|c|c|c|}
\hline \multicolumn{22}{|c|}{ AVALIAÇÃO DE PERIGOS E RISCOS } \\
\hline \multirow[t]{2}{*}{$\begin{array}{l}N \\
\underline{o}\end{array}$} & \multirow{2}{*}{$\begin{array}{l}\text { TAREFAS } \\
\text { LOCAL } \\
\text { EQUIPAMENT } \\
\text { OS }\end{array}$} & \multirow{2}{*}{ ঠొ } & \multirow{2}{*}{ 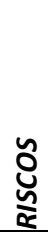 } & \multicolumn{4}{|c|}{$\begin{array}{l}\text { AVALIAÇÃO } \\
\text { SEM } \\
\text { CONTROLES }\end{array}$} & \multirow{2}{*}{$\begin{array}{l}\vec{K} \\
\frac{5}{0} \\
0 \\
\frac{n}{x}\end{array}$} & \multirow{2}{*}{$\begin{array}{l}\text { CONTROL } \\
\text { ES E } \\
\text { BARREIR } \\
\text { AS } \\
\text { EXISTENT } \\
\text { ES } \\
\end{array}$} & \multicolumn{4}{|c|}{$\begin{array}{l}\text { AVALIAÇÃO } \\
\text { COM } \\
\text { CONTROLES }\end{array}$} & \multirow{2}{*}{$\begin{array}{l}\frac{\pi}{x} \\
\frac{\pi}{0} \\
0 \\
\frac{\pi}{0}\end{array}$} & \multirow{2}{*}{$\begin{array}{l}\text { CONTROL } \\
\text { E OU } \\
\text { AÇÃO } \\
\text { REQUERI } \\
\text { DA }\end{array}$} & \multicolumn{4}{|c|}{$\begin{array}{l}\text { AVALIAÇÃO } \\
\text { COM } \\
\text { CONTROLES } \\
\text { ADCIONAIS }\end{array}$} & \multirow{2}{*}{ 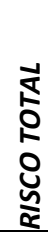 } & \multirow{2}{*}{$\begin{array}{l}\text { CONTROL } \\
E \quad O U \\
A C ̧ A ̃ O \\
\text { REQUERI } \\
\text { DA }\end{array}$} \\
\hline & & & & $\begin{array}{l}P \\
0 \\
\end{array}$ & \begin{tabular}{|l|}
$F$ \\
$E$ \\
\end{tabular} & $\begin{array}{l}\mathbf{P} \\
\mathbf{G}\end{array}$ & $\begin{array}{l}\mathrm{N} \\
\mathrm{P} \\
\end{array}$ & & & $\begin{array}{l}P \\
0\end{array}$ & \begin{tabular}{|l}
$F$ \\
$E$ \\
\end{tabular} & $\begin{array}{l}\text { P } \\
\text { G }\end{array}$ & $\begin{array}{l}\mathbf{N} \\
\mathbf{P}\end{array}$ & & & $\begin{array}{l}P \\
0\end{array}$ & \begin{tabular}{|l|}
$F$ \\
$E$ \\
\end{tabular} & $\begin{array}{l}\mathbf{P} \\
\mathbf{G}\end{array}$ & $\begin{array}{l}\mathbf{N} \\
\mathbf{P}\end{array}$ & & \\
\hline 1 & & & & & & & & 0 & & & & & & 0 & & & & & & 0 & \\
\hline 2 & & & & & & & & 0 & & & & & & 0 & & & & & & 0 & \\
\hline 3 & & & & & & & & 0 & & & & & & 0 & & & & & & 0 & \\
\hline
\end{tabular}

Documento necessário tanto para a execução de trabalhos com funcionários da empresa como de parceiros (Tabela 04). Mediante os dados e informações levantadas por meio de checklist e análise preliminar de risco, foi elaborada planilha para cálculo das variáveis definindo assim por meio da severidade, 
probabilidade, abrangência e frequência, o grau de risco da atividade elaborada pelo colaborador de manutenção.

\section{RESULTADOS E DISCUSSÃO}

Durante a realização do serviço ATR foi preenchida com todas as informações pertinentes a realização do trabalho, colhendo assinatura dos participantes e levando até o encarregado. Abaixo seguem os parâmetros apontados no estudo.

Tabela 5: ATR de manutenção da linha de água da centrifuga.

ATR Tipo de Trabalho

\begin{tabular}{|l|l|l|l|l|l|l} 
Quente & Químico & X & Altura & & Escavação
\end{tabular}

Equipamento de Proteção Individual Específico para o Trabalho

\begin{tabular}{|l|l|l|l|}
\hline & Óculos de Segurança & & Calçado de couro/ borracha \\
\hline & Luva raspa /látex/vaqueta & & Protetor auricular \\
\hline & Cinto de segurança & & \\
\hline & Capacete & & \\
\hline & Trava quedas & & \\
\hline & & & Outros: \\
\hline
\end{tabular}

Riscos Potenciais da Tarefa

\begin{tabular}{|l|l|l|l|}
\hline & Queimadura Térmica & & Queda \\
\hline & Choque elétrico & & Cortes/ perfurações \\
\hline & Vapores & & \\
\hline & Queimadura química & & \\
\hline & Partículas nos olhos & & Outros: \\
\hline & & Outros:
\end{tabular}

Checklist de Medidas de Controle - Preenchimento Obrigatório

\begin{tabular}{|l|l|l|l}
\hline $\mathrm{S}$ & NA & 1 - Trabalho a quente com risco de incêndio e explosão \\
\hline
\end{tabular}

\begin{tabular}{l|l|l}
$\mathrm{X}$ & Os equipamentos de corte/solda/furadeira estão em boas condições? \\
\hline
\end{tabular}

$\mathrm{X} \quad$ As mangueiras do conjunto oxicetilênico possui válvula de retrocesso de chama?

$\mathrm{X} \quad$ Existe a necessidade de molhar o local?

\begin{tabular}{l|l|lcl}
\hline & $\mathrm{X}$ & Há necessidade de instalação de tapume contra radiação e proteção de partículas?
\end{tabular}

\begin{tabular}{l|l|l}
$\mathrm{X}$ & O local foi isolado e sinalizado para impedir a passagem de pessoas estranhas ao serviço?
\end{tabular}

\begin{tabular}{|l|l|l|}
\hline & $\mathrm{X}$ & Os executantes de serviço a quente estão utilizando os EPIs adequados? \\
\hline & $\mathrm{X}$ & Os executantes são aptos e estão em condições físicas e mentais para executar o trabalho?
\end{tabular}

\begin{tabular}{l|l|l|l|l}
\hline & $\mathrm{X}$ & O local está limpo e livre de material combustível /inflamável /explosivo?
\end{tabular}

\begin{tabular}{l|l}
$\mathrm{X}$ & Há outro trabalho autorizado nas proximidades que possa influenciar a realização desta tarefa?
\end{tabular}

\begin{tabular}{l|l|lcl}
\hline & $\mathrm{X}$ & A área abaixo do serviço foi verificado, esta sinalizada, isolada e livre de material combustível?
\end{tabular}

\begin{tabular}{|l|l|l|}
\hline & $\mathrm{X}$ & Foi verificado a presença de gás inflamável em canaletas, poços, tubulações, etc? \\
\hline & $\mathrm{X}$ & Todas as fontes de energia foram etiquetadas, bloqueadas e travadas, conforme os procedimentos? \\
\hline
\end{tabular}

\begin{tabular}{ll|l}
\hline $\mathrm{X}$ & Os funcionários estão com cadeados de segurança nos suportes de travamento de energia?
\end{tabular}

\begin{tabular}{l|l|l|}
\hline$S$ & NA & 2 - Produtos químicos \\
\hline
\end{tabular}

\begin{tabular}{l|l|l|l}
\hline & $\mathrm{X}$ & Em casos de acidentes existe lava olhos nas proximidades?
\end{tabular}

\begin{tabular}{l|l}
$\mathrm{X}$ & O produto a descarregar é o mesmo contido no tanque a receber?
\end{tabular}

\begin{tabular}{|l|l|l}
\hline & $\mathrm{X}$ & Existe espaço no tanque para receber o produto? \\
\hline & $\mathrm{X}$ & O veículo está aterrado e o local isolado e sinalizado?
\end{tabular}

\begin{tabular}{l|l|l}
\hline $\mathrm{X}$ & As válvulas de drenagem dos diques de contenção estão fechadas? \\
\hline
\end{tabular}

\begin{tabular}{l|l|l}
\hline $\mathrm{X}$ & Existe balde/recipiente para conter pequenos vazamentos?
\end{tabular}

\begin{tabular}{|c|c|c|c|c|c|}
\hline & $\mathrm{X}$ & \multicolumn{4}{|l|}{ Os mangotes estão em boas condições de uso? } \\
\hline & $\mathrm{X}$ & \multicolumn{4}{|c|}{ O motorista permanecera junto ao local de descarga? } \\
\hline & $\mathrm{X}$ & \multicolumn{4}{|c|}{ Para descarga de solvente o portão de acesso está fechado e lâmpada de emergência está ativa? } \\
\hline & $\mathrm{X}$ & \multicolumn{4}{|c|}{ Os produtos químicos tem os rótulos com as informações sobre seus riscos? } \\
\hline & $\mathrm{X}$ & \multicolumn{4}{|c|}{ Existe ventilação eliminando gases/vapores com riscos a saúde e perigo de incêndio e explosões? } \\
\hline S & NA & \multicolumn{4}{|l|}{3 - Trabalho em altura } \\
\hline $\mathrm{X}$ & & Será necessário usar: & $\mathrm{X}$ & Andaime & 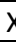 \\
\hline & $\mathrm{X}$ & \multicolumn{4}{|c|}{ Os andaimes/escadas estão nivelados e apoiados em solo firme? Rodas estão bloqueadas? } \\
\hline & $\mathrm{X}$ & \multicolumn{4}{|c|}{ Os montantes dos andaimes estão com travas diagonais e borboletas de fixação? } \\
\hline $\mathrm{X}$ & & \multicolumn{4}{|l|}{ Existe necessidade de amarrar o andaime? } \\
\hline
\end{tabular}




\begin{tabular}{|c|c|}
\hline $\mathrm{X}$ & Os pranchões estão resistentes, sem rachaduras e com travas nas extremidades? \\
\hline $\mathrm{X}$ & Serão utilizado cinto de segurança com duas cordas espias? \\
\hline $\mathrm{X}$ & Os cintos estão amarrados em locais resistentes, elevados e independente de andaime? \\
\hline $\mathrm{X}$ & Os cintos estão amarrados em locais resistentes, elevados e independente de andaime? \\
\hline $\mathrm{X}$ & Nos trabalhos em superfícies frágeis serão utilizadas pranchas/tabuas para distribuição de cargas? \\
\hline
\end{tabular}

Conforme os dados coletados da presente pesquisa o valor de HRN esteve entre 5 - 50 caracterizando nível de atenção, que apresenta risco em potencial. É recomendada cobrança severa da utilização de EPI e reciclagem dos funcionários quanto às atividades operacionais. O diagnóstico da análise vindica inspeção planejada do ambiente de trabalho para identificação de agentes agressivos (mecânico gravitacionais, cinético, elétrico, térmico), e garantia da eficácia das medidas atuais de proteção sendo aprimoradas com ações complementares.

A atividade carece da utilização de equipamentos de proteção individual (EPI) específico. São especificados e regulamentados através de norma regulamentadora NR6, destinada a proteção da saúde e segurança do trabalhador. Foram apontados os EPI específicos para o trabalho em questão: óculos de segurança, luvas (raspas, algodão, látex ou vaqueta), capacete, sinto de segurança, trava quedas, calçado de couro e protetor auricular.

No caso em que as informações do checklist não estiveram contempladas em sua totalidade da análise de risco, da obrigatoriedade de uso de EPI e das medidas de controle, estas foram inseridas manualmente em um formulário. Os riscos potenciais aliadas ao desenvolvimento da tarefa de finalizar a linha de água da centrifuga no setor biodiesel foram: queimaduras térmicas, choque elétrico, vapores, queimadura química, quedas, partículas nos olhos, cortes e perfurações.

A fim de evitar a ocorrência desses acidentes, em serviços a quente recipientes ou equipamentos em que existem a possibilidade de presença de vapores de combustíveis ou inflamáveis, deverá realizar avaliação com o medidor de gases, caso seja detectado a presença de gases o recipiente ou equipamento deve ser lavado, vaporizado e realizado nova avaliação.

\section{CONCLUSÕES}

Concluímos que para a realização das atividades descritas deve-se contar com profissional capacitado e treinado. Além disso, cabe aos trabalhadores cumprir com todas as orientações relativas ao processo, não realizar qualquer tipo de alteração nas proteções mecânicas, participar dos treinamentos fornecidos e comunicar ao gestor sobre possível dano no equipamento.

Para as medidas de controle deverão ser observados sempre àquelas citadas nas Normas de Segurança e Instruções de Segurança específicas de trabalho e dos setores. Deve haver acompanhamento constante da execução dos trabalhos priorizando por ordem crescente de risco e gravidade, exercendo ações para manter os riscos no mesmo status do momento da permissão, suspendendo a execução do trabalho em casos de alteração das condições de risco.

Todo serviço de manutenção mecânica, elétrica e civil deverá ser realizado com emissão de ATR, obedecendo todos os critérios estabelecidos de segurança em normas e instruções pertinentes. Todo 
trabalho (novo) não constante deverá ser submetido a análise de risco e ter aprovação conforme a sua condição de risco.

\section{REFERÊNCIAS}

ABNT. Associação Brasileira de Normas Técnicas. Norma Regulamentadora NR 6: Equipamento de proteção individual-EPI. Manual de legislação Atlas: Segurança e Medicina do Trabalho. 71 ed. São Paulo: Atlas, 2013.

ABNT. Associação Brasileira de Normas Técnicas. Norma Regulamentadora NR 12: Segurança no trabalho em máquinas e equipamentos. Manual de legislação Atlas Segurança e Medicina do Trabalho. 71 ed. São Paulo: Atlas, 2013.

BRASIL. Decreto Lei n. 5.452, de 10 de maio de 1943. Aprova a Consolidação das Leis do trabalho. Brasília: DOU, 1943.

BRASIL. Ministério do Trabalho e Emprego. Ministério da Previdência Social. Número de acidentes e doenças do trabalho no Brasil, de 1970 a 2008: acidentes de trabalho ocorridos nos últimos 39 anos. Brasília: MTE, 2009.

CARDELLA, B.. Segurança no trabalho e prevenção de acidentes: uma abordagem holística. Segurança integrada à missão organizacional com produtividade, qualidade, preservação ambienta e desenvolvimento de pessoas. São Paulo: Atlas, 2010.

CICCO, F.; FANTAZZINI, M. L.. Tecnologias consagradas de gestão de riscos. 2 ed. São Paulo: Risk Tecnologia, 2003.

DOONAN, P. F.. Fishing and risk management. Risk
Management, New York, v.48, n.12, p.48, 2001.

OMS. Organização Mundial da Saúde. Documento de referência da OMS sobre ambientes de trabalho saudáveis. Genebra, 2010.

FERREIRA, J. P.. Análise de riscos e contingência: Segurança, Meio Ambiente e Saúde (SMS) do Programa de Mobilização da Indústria Nacional de Petróleo e Gás Natural (PROMINP). 2007.

LAPA, R. P.; GOES, M. L. S.. Investigação e análise de incidentes Conhecendo o Incidente para prevenir. São Paulo: EDICON, 2011.

MAURO, M. Y. C.; MUZI, C. D.; GUIMARÃES, R. M.; MAURO, C. C. C.. Risco ocupacional em saúde. R. Enferm. UERJ, Rio de Janeiro, v.12, p.338-345, 2004.

OLIVEIRA, M. P... Um estudo de caso da gestão de segurança industrial de uma plataforma de petróleo offshore. Dissertação (Mestrado em Sistemas de Gestão) Universidade Federal Fluminense, Niterói, 2008.

PINTO, J. B.. Métodos de apreciação de risco de maquinas e equipamentos usados no brasil. Brasília, 2014.

SANTOS, R. R.. Adequação NR-12: Automação Industrial, Indústria 4.0. 2018.

A CBPC - Companhia Brasileira de Produção Científica (CNPJ: 11.221.422/0001-03) detém os direitos materiais desta publicação. Os direitos referem-se à publicação do trabalho em qualquer parte do mundo, incluindo os direitos às renovações, expansões e disseminações da contribuição, bem como outros direitos subsidiários. Todos os trabalhos publicados eletronicamente poderão posteriormente ser publicados em coletâneas impressas sob coordenação da Cognitionis Publishing, da Companhia Brasileira de Produção Científica e seus parceiros autorizados. Os (as) autores (as) preservam os direitos autorais, mas não têm permissão para a publicação da contribuição em outro meio, impresso ou digital, em português ou em tradução. 